\title{
Otimização Estocástica Dinâmica e Implícita para Operação de Reservatório: Estudo de Caso em Sergipe
}

\author{
Dynamic and Implicit Stochastic Optimization for Reservoir Operation: Case \\ Study in Sergipe
}

\author{
Alcigeimes B Celeste ${ }^{1}$, Raul Fontes Santana ${ }^{2}$ Wesley Medeiros Santos ${ }^{3}$ \\ 1,2,3 Universidade Federal de Sergipe, SE, Brasil \\ geimes@yahoo.com; eng.raulfs@gmail.com; wesley.medeiros87@gmail.com
}

\begin{abstract}
Resumo
Reservatórios de abastecimento de água são normalmente operados de acordo com as chamadas curvas-guia, que decidem a fração de demanda a ser atendida com base nas condições atuais. Este trabalho avalia a adequação da otimização estocástica implícita (OEI) perante a programação dinâmica estocástica (PDE) para configurar políticas de alocação de água. O estudo de caso é o maior reservatório do estado de Sergipe. Os modelos são aplicados para operar o reservatório sob vários cenários possíveis, cada um com 100 anos de afluências mensais. Operações são também conduzidas pela política de operação padrão de reservatórios $(S O P)$ e por otimização determinística considerando previsão perfeita de afluências futuras (ODPP), esta última utilizada como referência. Para todos os 100 cenários utilizados, o desempenho da PDE e da OEI é superior ao da SOP e próximo ao da ODPP. Além disso, o procedimento simples de OEI alcança performance similar à complexa técnica de PDE.
\end{abstract}

Palavras-chave: Reservatórios, otimização, estocasticidade, curvas-guia, operação.

\begin{abstract}
Water supply reservoirs are usually operated in accordance with so-called rule curves, which decide the ratio of demand to be met based on current conditions. This paper assesses the suitability of implicit stochastic optimization (ISO) against stochastic dynamic programming $(S D P)$ to set up reservoir release policies. The case study is the largest reservoir in the state of Sergipe, Brazil. The models are applied to operate the reservoir under several possible 100-year inflow scenarios. Operations are also carried out by the standard operating policy (SOP) of reservoirs and by deterministic optimization based on perfect inflow forecast (PFDO), the latter used as a benchmark. In all of the 100 scenarios utilized, the performance of both SPD and ISO is superior to that of SOP and close to that of PFDO. Furthermore, the simple ISO shows to perform similarly to the more complex SDP.
\end{abstract}

Keywords: Reservoirs, optimization, stochasticity, rule curves, operation. 


\section{Introdução}

Os problemas de abastecimento de água, com demandas gradativamente maiores e longos períodos de seca, estão exigindo cada vez mais dos reservatórios existentes, ocorrendo até mesmo casos de utilização de seus volumes mortos, como o que vem acontecendo durante os últimos meses no Sistema Cantareira (São Paulo). Os gestores de recursos hídricos são, desta forma, pressionados a utilizarem políticas e metodologias mais eficientes na operação dos reservatórios.

Na prática, a operação de reservatórios é guiada pelas chamadas curvas-guia, que estabelecem a quantidade de vazão a ser liberada em função do armazenamento atual e, eventualmente, da previsão de afluências futuras. Curvas-guia são tipicamente construídas a partir de modelos de simulação. No entanto, como sempre existe uma quantidade muito grande de possibilidades de operação, o procedimento para encontrar a melhor delas é bastante trabalhoso e nem sempre a opção adotada é a melhor.

Outra alternativa para conduzir a operação de um reservatório é por meio de métodos de otimização matemática, os quais verificam implicitamente todas as possibilidades e automaticamente identificam a opção ótima (Ahmad et al., 2014; Farhangi et al., 2012; Rani e Moreira, 2010; Labadie, 2004). O problema dessa alternativa está relacionado com as incertezas hidrológicas, tais como as das afluências futuras, para as quais geralmente são utilizados valores médios históricos, previsões, ou cenários futuros possíveis (otimização por cenários).

De acordo com Celeste (2011), a incerteza das vazões afluentes torna-se importante quando os valores médios históricos não são suficientes para representar adequadamente as condições hidrológicas altamente variáveis ou quando as afluências não podem ser previstas de forma confiável por um período relativamente longo. Em tais casos, o problema é tipicamente tratado pela programação dinâmica estocástica (PDE), um tipo de otimização estocástica explícita (OEE) que incorpora métodos probabilísticos de vazão diretamente no problema de otimização (Barros, 1997). No entanto, procedimentos de OEE apresentam dificuldade de aceitação pelos operadores, em virtude dos cálculos matemáticos avançados que envolvem e da dificuldade de implementação em relação a modelos simples de simulação.

Como maneira de intermediar modelos de simulação (simples) com métodos de otimização (complexos), existe uma ferramenta denominada de otimização estocástica implícita (OEI) que, além de ser capaz de incorporar a estocasticidade das vazões afluentes e de fornecer curvas-guia operacionais, também são mais fáceis de serem compreendidas (Celeste e Billib, 2012, 2009; Celeste et al., 2009).

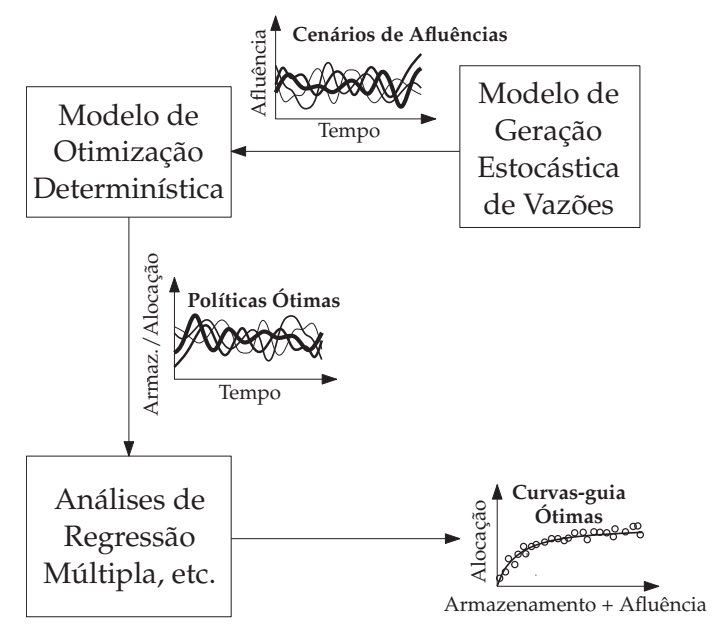

Figura 1: Otimização Estocástica Implícita.

Neste trabalho, a técnica de otimização estocástica implícita é aplicada para derivar regras operacionais ótimas tendo como estudo de caso o reservatório formado pela recém construída barragem do rio Poxim, no estado de Sergipe. As regras operacionais da OEI são comparadas com operações realizadas pela chamada política de operação padrão, por programação dinâmica estocástica, e por um modelo de otimização determinística considerando previsão perfeita de afluências.

\section{Material e Métodos}

\subsection{Otimização Estocástica Implícita}

A ideia básica da otimização estocástica implícita, também denominada de otimização Monte Carlo (Farias et al., 2006), é empregar um modelo de otimização determinística a fim de encontrar as alocações ótimas do reservatório para vários cenários distintos de vazões afluentes. Para cada uma das realizações de afluência, uma política de operação diferente é encontrada. O conjunto de todas as políticas operacionais é então examinado de forma a construir regras de alocação para o reservatório (ver Figura 1).

Neste trabalho, o procedimento de OEI utilizado para a construção de regras mensais foi conduzido da seguinte forma:

1. Gerar 100 cenários, cada um com 110 anos de afluências mensais (Seção 2.1.1);

2. Para cada sequência de 110 anos, achar as liberações ótimas de água a partir de um modelo de otimização determinística sob previsão perfeita de afluências (Seção 2.1.2). Descartar os primeiros e últimos cinco anos de dados para evitar a influência de condições iniciais e finais de armazenamento; 
3. Subdividir o conjunto obtido de dados operacionais ótimos em subconjuntos para cada mês do ano (janeiro até dezembro);

4. Para cada subconjunto contendo um total de 10000 dados (100 cenários $\left.\times 100 \frac{\text { anos }}{\text { cenário }}\right)$, correlacionar a alocação de cada mês $t$ do ano, $R(t)$, com o volume armazenado no início do mês, $S(t-1)$, e a afluência esperada para o mês, $I(t)$, da mesma forma como é feito usualmente (Labadie, 2004):

$$
R(t)=\psi(S(t-1), I(t))
$$

Tipicamente, técnicas de regressão linear e não linear via polinômios são empregadas para gerar a curva da função $\psi$ que correlaciona as variáveis. Esta pesquisa utilizou outro tipo de procedimento detalhado na Seção 2.1.3.

\subsubsection{Modelo de Geração de Afluências Sintéticas}

Os diversos cenários de afluências necessários no passo 1 do procedimento acima podem ser obtidos a partir de registros históricos (se houver disponibilidade de séries suficientemente longas) mas são usualmente gerados pela extensão da série histórica existente através de modelos de geração estocástica de vazões. Tais modelos devem preservar as propriedades estatísticas dos dados históricos (média, desvio padrão, autocorrelação, etc.) nos dados sintéticos.

Este trabalho utilizou o modelo de Thomas-Fiering (Thomas e Fiering, 1962), verificado em outras pesquisas como sendo um procedimento eficiente para geração sintética de vazão em rios nordestinos, particularmente rios sergipanos (Celeste et al., 2012, 2009, 2006).

\subsubsection{Modelo de Otimização Determinística}

O modelo de otimização determinística, utilizado no passo 2 do procedimento de OEI adotado, assume que o principal objetivo da operação é satisfazer as demandas o máximo possível sem comprometer o sistema, encontrando as alocações ótimas mensais de água do reservatório. O objetivo é minimizar a soma dos desvios quadráticos entre liberações e demandas sujeito à equação do balanço hídrico do reservatório e a restrições de domínio, que definem limites inferiores e superiores para armazenamentos, liberações e vertimentos. $\mathrm{O}$ problema de otimização é formulado como segue:

$$
\operatorname{minimizar} \sum_{t=1}^{N}\left[\frac{D(t)-R(t)}{D(t)}\right]^{2}
$$

sujeito a

$$
\begin{gathered}
S(t)=S(t-1)+I(t)-E(t)-R(t)-S p(t), \quad \forall t \\
0 \leq R(t) \leq D(t), \quad \forall t
\end{gathered}
$$

$$
\begin{gathered}
S_{\min } \leq S(t) \leq S_{\max } \quad \forall t \\
S p(t) \geq 0, \quad \forall t
\end{gathered}
$$

onde $N$ é o horizonte de operação em meses; $R(t)$ e $D(t)$ são, respectivamente, alocação e demanda no mês $t ; I(t)$ e $E(t)$ são, respectivamente, o volume afluente ao reservatório e o volume evaporado no mês $t$; $S(t)$ é o volume do reservatório no final do mês $t ; S(t-1)$ é o volume do reservatório no final do mês anterior $t-1 ; S_{\max }$ é a capacidade máxima de armazenamento do reservatório; $S_{\min }$ é o volume morto do reservatório; e $S p(t)$ é o volume de vertimento. A função objetivo (2) é o somatório dos quadrados das taxas de vulnerabilidade $\alpha(t)=[D(t)-R(t)] / D(t)$ ao longo de todos os meses $t$ do horizonte operacional de $N$ meses.

Utilizando um procedimento de linearização da curva área-volume do reservatório para o cálculo da evaporação, esse modelo torna-se um problema de programação quadrática (PQ), no qual a função objetivo é quadrática e as restrições são funções lineares. Celeste e Billib (2010) propuseram resolver este modelo de modo que não ocorra vertimento quando o nível de armazenamento no reservatório estiver menor que sua capacidade.

Para um cenário pré-definido de afluências, i.e., com valores de $I(t)$ conhecidos para todos os meses $t=$ $1, \ldots, N$, tem-se um modelo de otimização determinística sob previsão perfeita (ODPP).

\subsubsection{Interpolação das Curvas-Guia}

O modelo de OEI primeiramente agrupa por mês os dados operacionais resultantes da otimização determinística. Para isso, a liberação é condicionada ao armazenamento do reservatório no início do mês e à afluência para o dado mês (Equação (1)). Ao invés de ajustar equações de regressão, como é feito na maioria das formas de OEI, este trabalho ajustou superfícies bidimensionais aos dados de liberação vs. armazenamento inicial e afluência mensal. Por causa disso, o modelo foi chamado de OEI-SURF.

A modelagem das superfícies foi realizada por meio de uma rotina de ajuste chamada de gridfit e encontrada em domínio público (D’Errico, 2005). Seu objetivo é construir uma superfície suave que aproxima seus pontos, porém permitindo o controle do nível de suavidade. A rotina é capaz de manipular dados com repetições e pontos colineares bem como extrapolar para além dos dados.

\subsection{Estudo de Caso}

O reservatório utilizado como estudo de caso desta pesquisa é formado pela barragem do rio Poxim, localizada no município de São Cristóvão, Sergipe. O reservatório foi inaugurado em 2013, tornando-se o maior do estado 


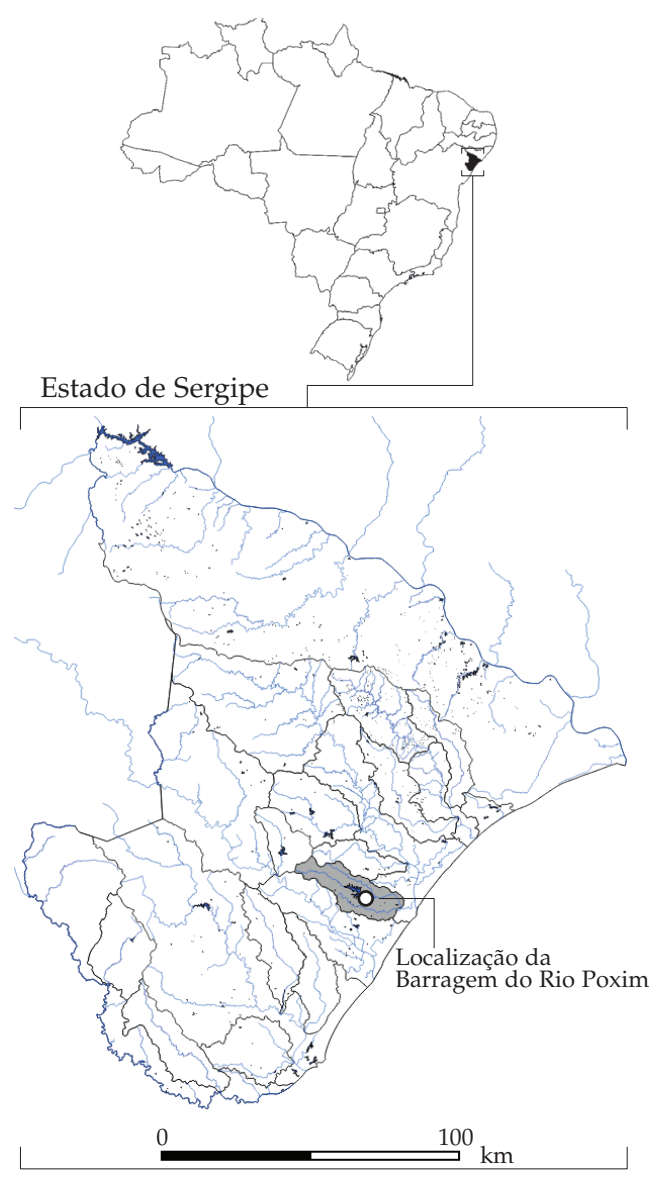

Figura 2: Localização da barragem do rio Poxim na subbacia hidrográfica do rio Poxim (Fonte: Base de Dados Geoespacial de Sergipe (SEMARH-SE)).

com capacidade de acumular até 32 milhões de metros cúbicos de água (ver Figura 2).

A demanda mensal de água foi assumida como sendo a vazão média de longo período (MLP) de 1,56 $\mathrm{m}^{3} / \mathrm{s}$. Essa demanda corresponde a uma garantia de atendimento de apenas $74 \%$, mas foi assumida porque era desejado observar várias situações de escassez e comparar como os modelos se comportariam perante as mesmas.

\subsection{Política de Operação Padrão}

Uma das regras operacionais mais simples é a política de operação padrão de reservatórios (SOP: standard operating policy), que condiciona a liberação de água à disponibilidade hídrica. A SOP especifica a vazão defluente total do reservatório em função da água disponível (armazenamento útil + afluência - evaporação). Quando a água disponível é menor que a demanda, todo o armazenamento é liberado e a demanda não é atendida por completo. Do contrário, o excesso de água é armazenado até que vertimento ocorra (ver Figura 3).

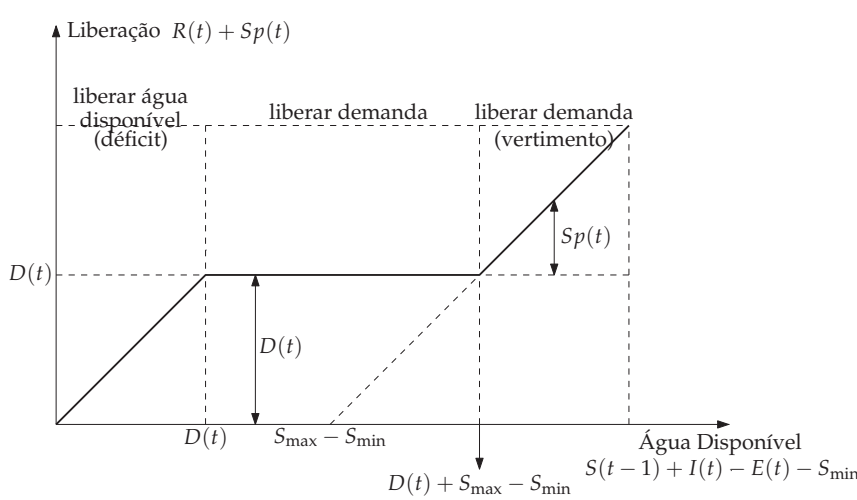

Figura 3: Política de operação padrão (SOP).

\subsection{Programação Dinâmica Estocástica}

A operação de reservatórios precisa levar em conta a aleatoriedade das vazões afluentes futuras. A programação dinâmica estocástica (PDE) é a técnica mais utilizada para operar reservatórios perante incertezas. A PDE incorpora a distribuição de probabilidades das vazões afluentes históricas diretamente no problema de otimização.

Neste trabalho, a função recursiva (Barros, 1997) do modelo de PDE utilizada foi:

$$
\begin{aligned}
F_{t}^{n}(S(t-1), I(t))= & \operatorname{minimizar}_{S(t) \text { viável }}[\alpha(t)+ \\
& \left.\sum_{I(t+1)} P_{I(t+1)} F_{t+1}^{n-1}(S(t), I(t+1))\right]
\end{aligned}
$$

onde $t$ é o mês atual e $n$ é o número total de meses remanescentes (até o horizonte $N$ ). $\mathrm{O}$ armazenamento inicial $S(t-1)$ e a afluência atual $I(t)$ são as variáveis de estado, enquanto que o armazenamento final $S(t)$ é a variável de decisão. $\alpha(t)$ é a taxa de vulnerabilidade no mês $t$ e $P_{I(t+1)}$ é a probabilidade incondicional da vazão afluente (sem correlação entre afluências consecutivas).

Para a calibração do modelo de PDE, as afluências históricas e o armazenamento foram discretizados em 100 valores espaçados igualmente do mínimo até o máximo. Para as afluências, o tamanho de cada classe foi obtido fazendo-se a segunda afluência discreta menos a primeira (ver Figura 4). O limite superior da primeira classe foi assumido como $75 \%$ da afluência mínima e seu limite inferior igual a zero. Já o limite superior da segunda classe foi dado pela afluência mínima mais o tamanho da classe dividido por 2. Os demais limites superiores das classes foram obtidos pelos limites superiores das classes anteriores mais o tamanho da classe, sendo que a classe final não possui um limite superior. A primeira classe da variável de estado armazenamento teve limite inferior igual a $S_{\min }$ e a última classe teve limite superior igual a $S_{\max }$ como mostrado na Figura 4. 


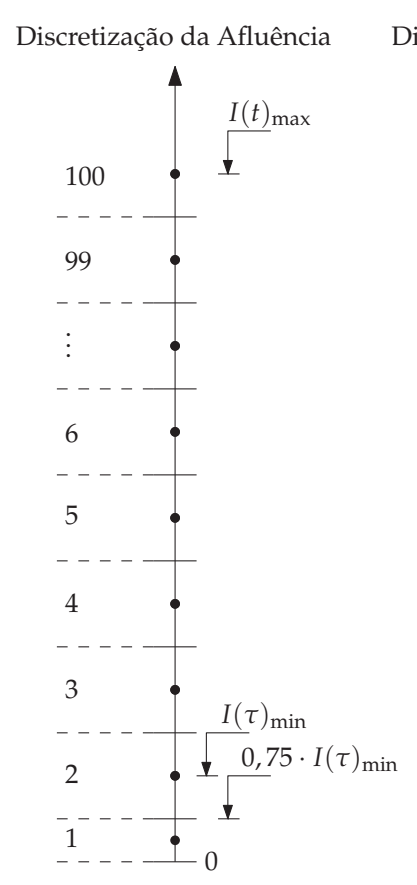

Discretização do Amazenamento

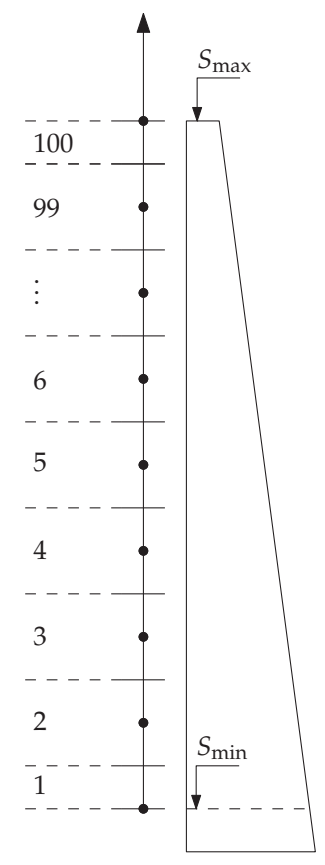

Figura 4: Discretização usada pelo modelo de PDE para cada mês $\tau=1, \ldots, 12$ (janeiro até dezembro). Os números indicam as classes e os pontos ao longo dos eixos verticais representam os valores discretos.

\subsection{Validação dos Modelos}

A fim de validar os modelos OEI-SURF e PDE, outros 100 conjuntos de 110 anos de afluências mensais foram gerados. Após a sua definição, as curvas-guia dos modelos foram aplicadas para operar o reservatório sob esses cenários. Para comparação, a SOP e a ODPP também foram utilizadas para operar o sistema perante as mesmas séries. A operação do sistema em face da previsão perfeita fornece as alocações ideais que deveriam ser empregadas para todos os meses do horizonte operacional de 110 anos, uma vez que, nesta situação, o modelo tem conhecimento de todos os influxos futuros mensais. Assim, as soluções da ODPP foram usadas como referência. $\mathrm{O}$ índice abaixo, igual a vulnerabilidade média quadrática ao longo de todos os $N$ meses de operação, foi utilizado para comparar as diferentes abordagens:

$$
\alpha=\frac{1}{N} \sum_{t=1}^{N}[\alpha(t)]^{2}=\frac{1}{N} \sum_{t=1}^{N}\left[\frac{D(t)-R(t)}{D(t)}\right]^{2}
$$

Novamente, como na calibração do modelo OEISURF, os resultados dos primeiros e últimos cinco anos foram descartados de forma que, para cada cenário, $N=100$ anos $\times 12 \frac{\text { meses }}{\text { ano }}=1200$ meses.

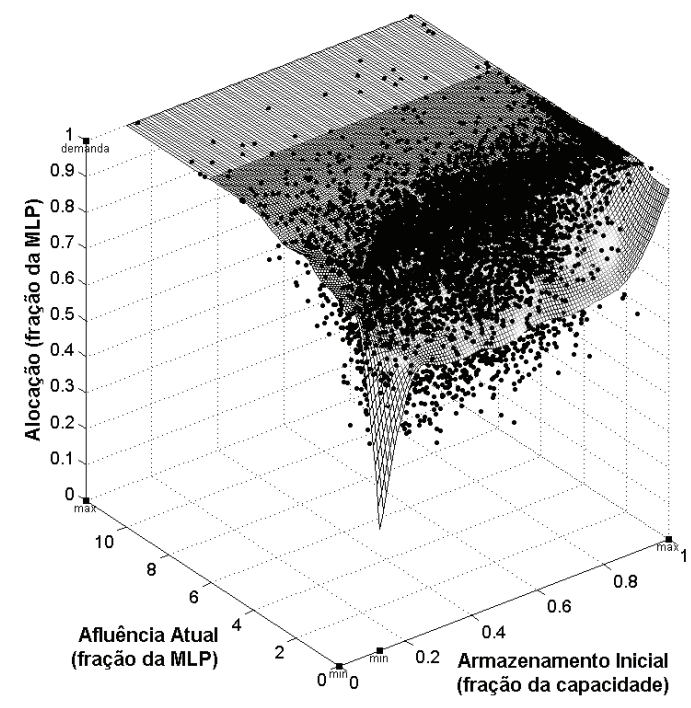

Figura 5: Superfície ajustada aos dados operacionais ótimos do mês de julho pelo modelo OEI-SURF.

\section{Resultados e Discussão}

A Figura 5 mostra um exemplo da superfície ajustada aos dados operacionais ótimos do mês de julho pelo modelo OEI-SURF. As superfícies para os outros meses foram semelhantes (Santana, 2015). É possível notar grande dispersão nos dados ótimos, significando que a liberação não é função apenas do armazenamento inicial e da afluência do mês. Contudo, a correlação destas três variáveis apenas deixa o modelo bastante simples e eficiente, como será discutido adiante.

Os valores de vulnerabilidades (Equação 3) para todas as séries de validação são mostrados na Figura 6 . Para exemplificar umas destas operações, os resultados para um dos 100 cenários são mostrados nas Figuras 7-10.

A Figura 7 mostra que a SOP, embora satisfaça grande parte da demanda, possui alta vulnerabilidade nos períodos de seca visto que não considera reter água para minimizar o efeito da escassez. De forma a mitigar os déficits extremos de água, a ODPP (ver Figura 8) é capaz de prever as secas e restringir a liberação de água através de racionamento. Isso é, de fato, o objetivo da ODPP (ver função objetivo (2)), ou seja, minimizar a vulnerabilidade total. A PDE e a OEI-SURF também têm como objetivo minimizar a vulnerabilidade e, como esperado, apresentaram um desempenho melhor do que o da SOP (ver Figuras 9 e 10, respectivamente).

Considerando a média das vulnerabilidades obtidas para todos os cenários, observou-se que a SOP apresentou-se em torno de 185,02\% mais vulnerável do que a ODPP, enquanto que, para os modelos PDE e OEISURF, essa taxa foi de $56,57 \%$ e $57,32 \%$, respectivamente.

Um aspecto importante a se observar é o fato de 


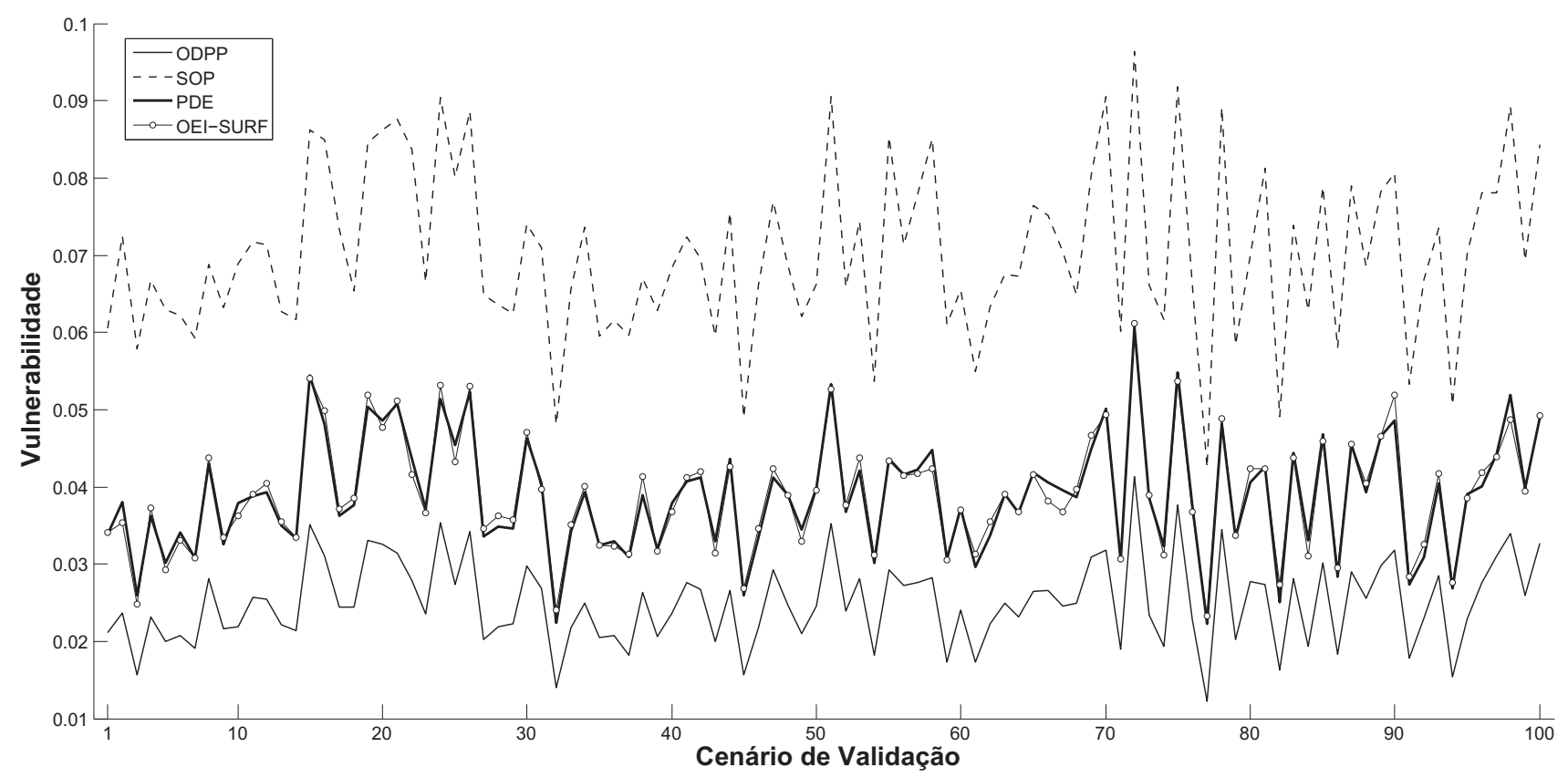

Figura 6: Comparação de vulnerabilidades para todos os modelos e cenários de validação.

os modelos OEI-SURF e PDE terem apresentado resultados muitos próximos, como observado na Figura 6. Devido à complexidade matemática da $\mathrm{PDE}$, muitos operadores de reservatório preferem usar estratégias de simulação, que são de fácil compreensão. Os resultados deste estudo mostram que a OEI-SURF pode ser uma boa alternativa, uma vez que é um modelo simples que produz regras de liberação equivalentes as geradas pela PDE.

\section{Conclusões}

Este artigo apresentou uma comparação de um modelo de otimização estocástica implícita (OEI-SURF) com outro de otimização estocástica explicita (PDE) aplicados para a operação de um reservatório localizado no estado de Sergipe. A política operacional padrão (SOP) também foi usada para comparação e operações geradas por otimização determinística sob previsão perfeita de vazões futuras foram tomadas como ideais. As regras mensais desenvolvidas por todos os modelos foram aplicadas para operar o sistema perante 100 cenários distintos de afluências e a vulnerabilidade de cada modelo foi medida para todos os cenários.

Os resultados mostraram que a PDE e a OEI-SURF obtiveram desempenho melhor do que a SOP. Além disso, a OEI-SURF forneceu regras de alocação semelhantes às encontradas por PDE. Isto é interessante porque, dada a sua simplicidade, este modelo pode ser mais aceitável por parte dos operadores de reservatórios quando comparado com otimização estocástica explícita.
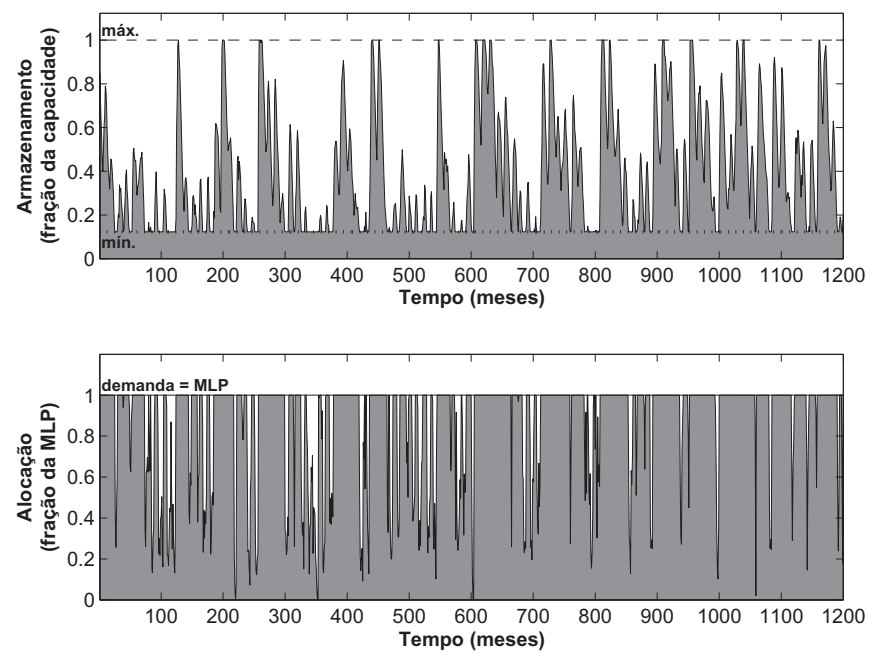

Figura 7: Operação do reservatório via SOP.

\section{Agradecimentos}

O primeiro autor agradece ao Conselho Nacional de Desenvolvimento Científico e Tecnológico (CNPq) pelo apoio financeiro. 

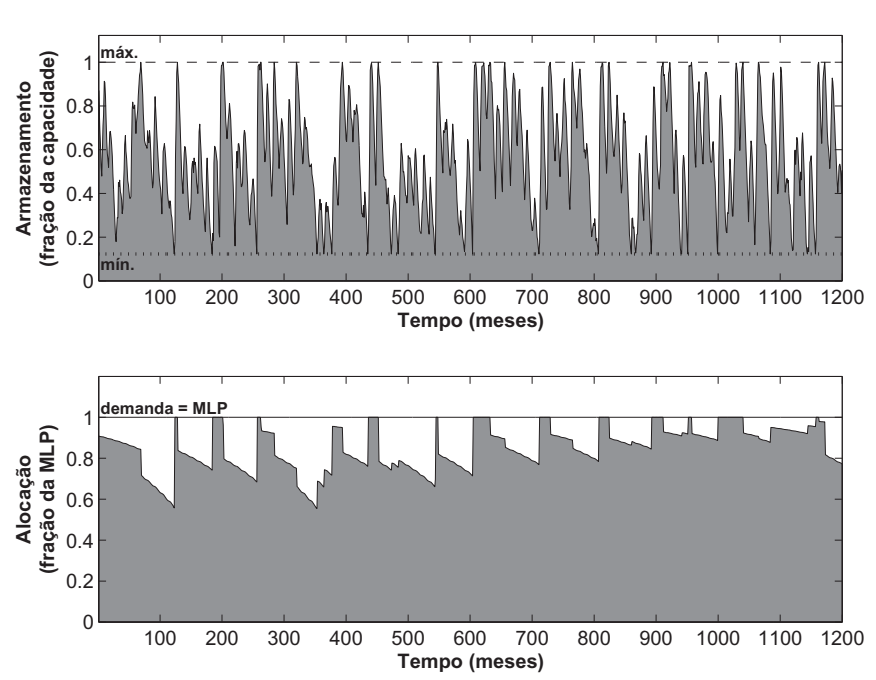

Figura 8: Operação do reservatório via ODPP.
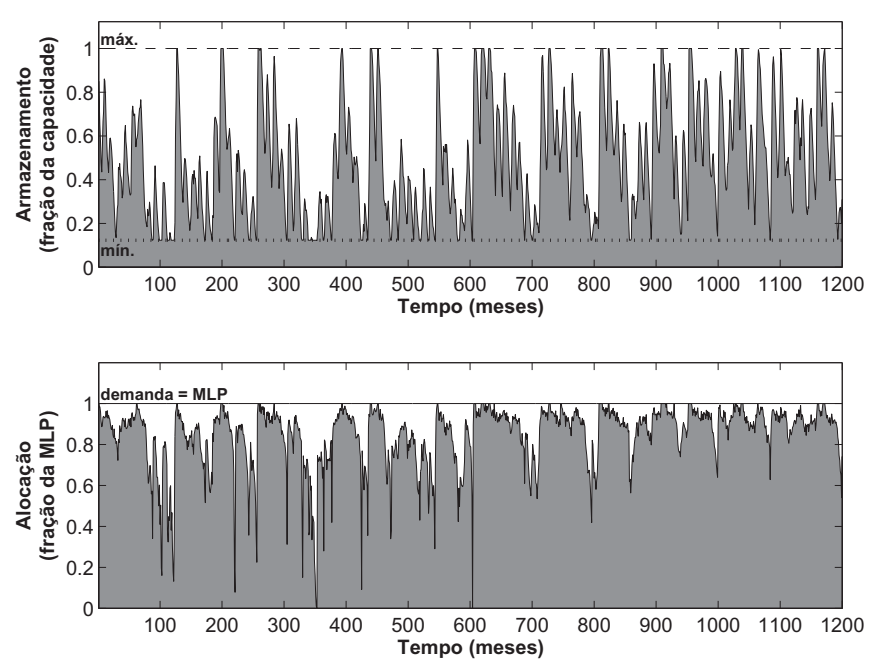

Figura 9: Operação do reservatório via PDE.
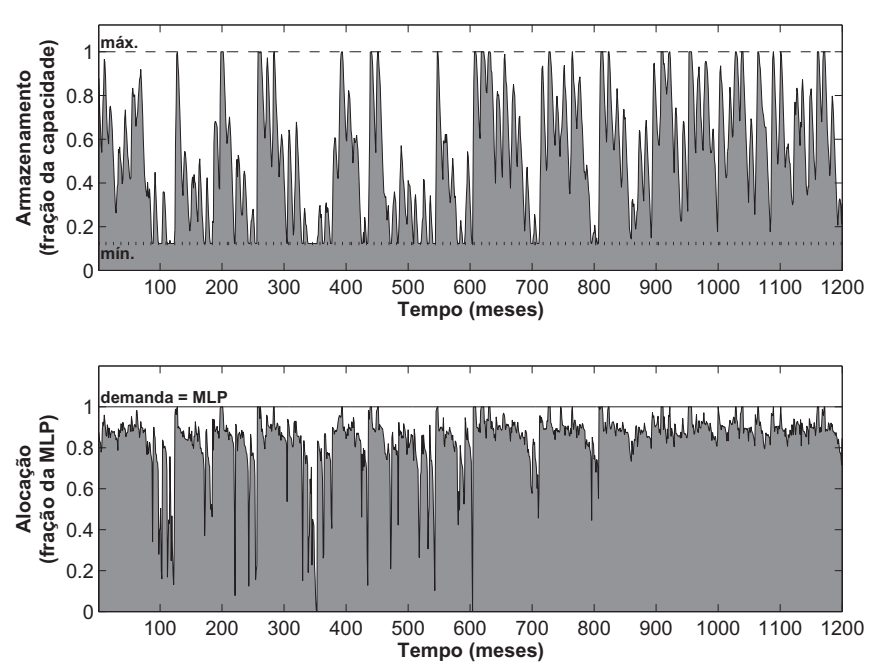

Figura 10: Operação do reservatório via OEI-SURF.

\section{Referências}

AHMAD, A., EL-SHAFIE, A., RAZALI, S. F. M., MOHAMAD, Z. S., 2014. Reservoir optimization in water resources: a review. Water Resources Management 28 (11), 3391-3405.

BARROS, M. T. L., 1997. A programação dinâmica aplicada à engenharia de recursos hídricos. In: Porto, R. L. L., et al. (Eds.), Técnicas Quantitativas para o Gerenciamento de Recursos Hídricos. Editora Universidade/ UFRGS/ABRH, Porto Alegre, pp. 239-277.

CELESTE, A. B., 2011. Comparação de modelos de otimização estocástica implícita e explícita na derivação de regras operacionais mensais de reservatórios. In: XIX Simpósio Brasileiro de Recursos Hídricos. Associação Brasileira de Recursos Hídricos (ABRH), Maceió.

CELESTE, A. B., BILLIB, M., 2009. Evaluation of stochastic reservoir operation optimization models. Advances in Water Resources 32 (9), 1429-1443.

CELESTE, A. B., BILLIB, M., 2010. The role of spill and evaporation in reservoir optimization models. Water Resources Management 24 (4), 617-628.

Celeste, A. B., Billib, M., 2012. Improving implicit stochastic reservoir optimization models with long-term mean inflow forecast. Water Resources Management 26 (9), 2443-2451.

CELESTE, A. B., CURI, W. F., CURI, R. C., 2006. Derivação de regras para a operação de reservatórios perante incertezas. In: VIII Simpósio de Recursos Hídricos do Nordeste. Associação Brasileira de Recursos Hídricos (ABRH), Gravatá, PE.

CELESTE, A. B., CURI, W. F., CURI, R. C., 2009. Implicit stochastic optimization for deriving reservoir operating rules in semiarid Brazil. Pesquisa Operacional 29 (1), 223-234.

CELESTE, A. B., FEITOSA, D. T., CORREIA, E. M., 2012. Análise de métodos para geração de vazões sintéticas no agreste sergipano. In: XI Simpósio de Recursos Hídricos do Nordeste. Associação Brasileira de Recursos Hídricos (ABRH), João Pessoa.

D'ERRICO, J., 2005. Surface fitting using gridfit. URL http://www.mathworks.com/matlabcentral/ fileexchange/8998

FARHANGI, M., BOZORG-HADDAD, O., MARIÑ, M. A., 2012. Evaluation of simulation and optimisation models for WRP with performance indices Proceedings of the Institution of Civil Engineers - Water 
Management 165 (5), 265-276.

FARIAS, C. A. S., CELESTE, A. B., SAKATA, Y., KADOTA, A., SUZU KI, K., 2006. Use of Monte Carlo optimization and artificial neural networks for deriving reservoir operating rules. Annual Journal of Hydraulic Engineering - JSCE 50, 25-30.

LABADIE, J. W., 2004. Optimal operation of multireservoir systems: State-of-the-art review. Journal of Water Resources Planning and Management ASCE 130 (2), 93-111.

RANI, D., MOREIRA, M. M., 2010. Simulationoptimization modeling: A survey and potential application in reservoir systems operation. Water Resources Management 24 (6), 1107-1138.

SANTANA, R. F., 2015. Operação de reservatório via otimização estocástica implícita: Estudo de caso em Sergipe. Trabalho de Conclusão de Curso (Engenharia Civil), Universidade Federal de Sergipe, São Cristóvão -SE.

THOMAS, H. A., FIERING, M. B., 1962. Mathematical synthesis of stream-flow sequences for the analysis of river basins by simulation. In: Maass, A., et al. (Eds.), Design of Water Resource Systems. Harvard Univ. Press, Cambridge, Massachusetts, pp. 459-493. 